\title{
Construction de cartes mentales synthétiques : mise en avant des représentations spatiales collectivement partagées
}

Le cas des travailleurs créatifs de quartiers urbains centraux de Nantes et Marseille

\section{Basile Michel}

\section{OpenEdition}

\section{Journals}

Édition électronique

URL : https://journals.openedition.org/cdg/1341

DOI : $10.4000 / \mathrm{cdg} .1341$

ISSN : 2107-7266

\section{Éditeur}

UMR 245 - CESSMA

\section{Référence électronique}

Basile Michel, «Construction de cartes mentales synthétiques : mise en avant des représentations spatiales collectivement partagées », Carnets de géographes [En ligne], 11 | 2018, mis en ligne le 15 septembre 2018, consulté le 20 mai 2021. URL : http://journals.openedition.org/cdg/1341 ; DOI : https://doi.org/10.4000/cdg.1341

Ce document a été généré automatiquement le 20 mai 2021.

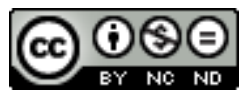

La revue Carnets de géographes est mise à disposition selon les termes de la Licence Creative Commons Attribution - Pas d'Utilisation Commerciale - Pas de Modification 4.0 International. 


\section{Construction de cartes mentales synthétiques : mise en avant des représentations spatiales collectivement partagées}

Le cas des travailleurs créatifs de quartiers urbains centraux de Nantes et Marseille

Basile Michel

\section{Introduction}

1 Dans le cadre de ma thèse en géographie, j'ai recueilli et analysé les représentations spatiales de travailleurs créatifs (artistes, designers...) implantés dans d'anciens quartiers populaires de Nantes et Marseille. Ces représentations sont définies comme la reconstruction intellectuelle des réalités spatiales perçues et imaginées à travers les filtres cognitifs et sociaux (Depeau, 2006). Depuis les travaux de Kevin Lynch (1960), la carte mentale fait partie des outils mobilisés pour saisir les représentations spatiales individuelles (Gieseking, 2013 ; Moser et Weiss, 2003). Elle est définie comme une " expression cartographique d'une représentation subjective de l'espace " (Lévy et Lussault, 2003: 132). Elle permet de mettre en évidence les éléments constitutifs de l'espace vécu des individus (Frémont, 1999). La difficulté qui accompagne son interprétation rend nécessaire l'utilisation précautionneuse de cet outil et sa combinaison avec d'autres techniques tels que l'entretien (Morange et al., 2016).

2 Si les représentations exprimées à travers la carte mentale sont propres à chaque individu, des éléments communs peuvent émerger à l'échelle des groupes sociaux (Roulier, 2013). L'identification et l'étude de ces éléments communs permettent de saisir plus en profondeur le « signifiant spatial » et « le signifié culturel » (Bailly, 1990 : 11). La question de la méthodologie permettant la mise en évidence et l'analyse des éléments collectivement partagés dans les représentations spatiales d'un groupe social 
se pose alors. Comment dépasser l'échelle individuelle d'analyse des représentations spatiales pour s'intéresser aux éléments qui font sens au niveau collectif? Dans l'objectif d'avancer sur cette question, cet article est centré sur la démarche méthodologique suivie pour construire des cartes mentales synthétiques à partir d'enquêtes sur les représentations spatiales de travailleurs créatifs regroupés au sein de quartiers urbains. Ces cartes mentales synthétiques visent à produire une image du quartier tel qu'il est défini dans les représentations de l'ensemble des travailleurs.

3 Cet article est structuré en deux parties. La première présente les terrains enquêtés et la démarche méthodologique utilisée pour construire les cartes mentales synthétiques. La deuxième s'articule autour des trois principaux résultats tirés de l'analyse de ces cartes.

\section{La carte mentale synthétique : un outil d'analyse des représentations spatiales des travailleurs créatifs}

4 Cette première partie présente les deux terrains choisis pour cette recherche avant de détailler la méthodologie mise en place pour construire les cartes mentales synthétiques.

\section{Le quartier source et objet de représentations collectivement partagées par les travailleurs créatifs ? Enquêtes dans deux quartiers urbains centraux}

5 Cet article s'appuie sur des travaux de recherche menés auprès de travailleurs des secteurs culturels et créatifs ayant leur bureau ou leur atelier professionnel dans les quartiers des Olivettes (Nantes) et du Panier (Marseille) ${ }^{1}$. Ces deux anciens quartiers populaires proches du centre-ville ont connu un déclin démographique et économique dans la seconde moitié du XXème siècle (Attard-Maraninchi, 1997; Petiteau, 2012). A partir des années 1990, leur progressive rénovation par les pouvoirs publics s'accompagne de l'installation de travailleurs créatifs. Ces derniers sont attirés par la centralité, les prix de l'immobilier abordables, les qualités sensibles (traces de l'histoire ouvrière préservées...) et les dynamiques créatives de ces quartiers. Ainsi, les Olivettes et le Panier concentrent aujourd'hui de nombreuses entreprises et associations appartenant aux secteurs culturels et créatifs ${ }^{2}$ (Figures 1 et 2 ).

6 A l'heure des échanges numériques et des mobilités accrues, l'agglomération spatiale des travailleurs créatifs incite à interroger les liens symboliques qu'ils entretiennent avec leur quartier d'implantation professionnelle ${ }^{3}$. Cet espace existe-t-il dans leurs représentations ? Et si oui, y-a-t-il une représentation du quartier collectivement partagée par l'ensemble de ces travailleurs? 
Figure 1. Carte du quartier des Olivettes

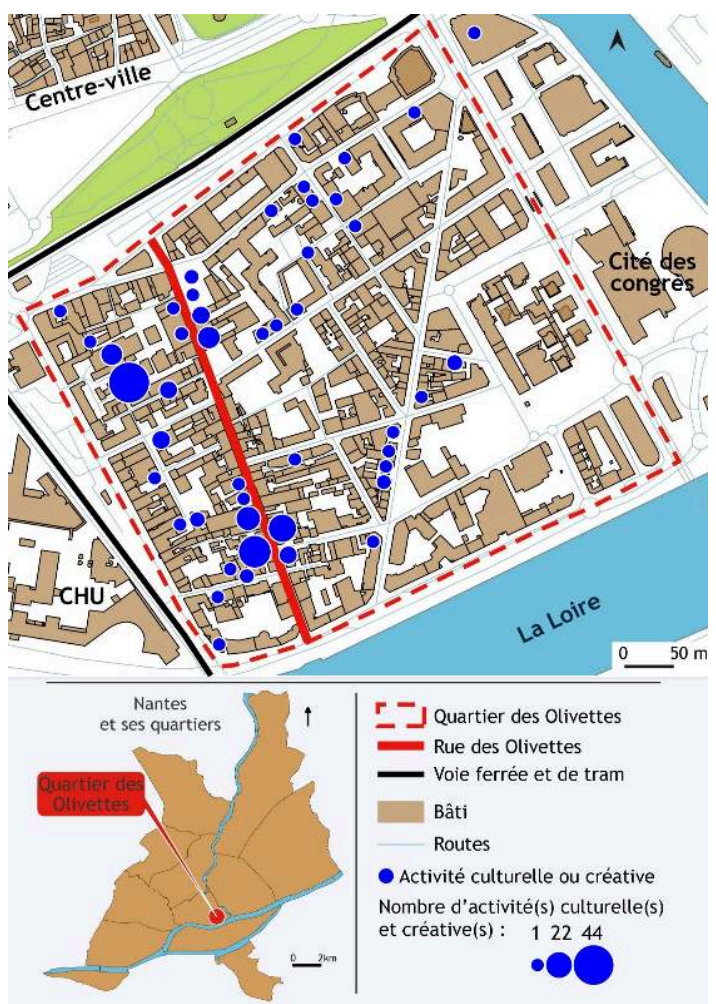

Source : Michel, 2018

Figure 2. Carte du quartier du Panier

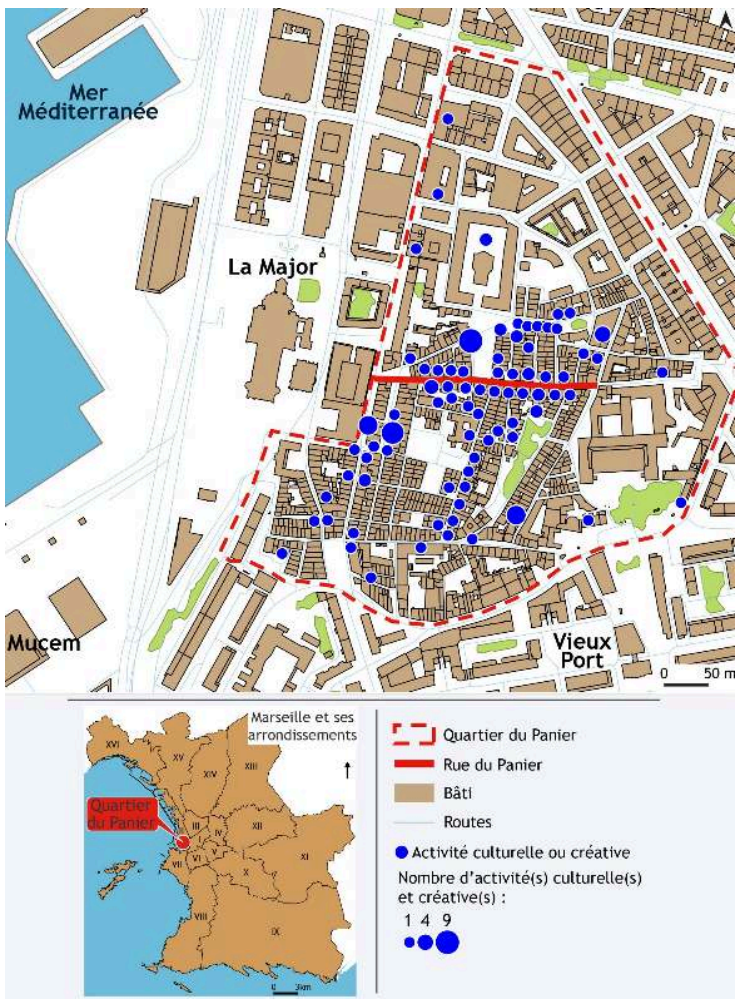

Source : Michel, 2018 


\section{Construction de cartes mentales synthétiques}

7 Pour identifier et analyser les éléments collectivement partagés par les travailleurs créatifs en termes de représentations de leur quartier d'implantation professionnelle, la démarche méthodologique mise en place a suivi trois étapes.

8 La première correspond au recueil des représentations spatiales individuelles. Pour cela, des entretiens semi-directifs ont été réalisés en 2015 et 2016 auprès de 102 travailleurs créatifs ${ }^{4}$ choisis de manière à obtenir un échantillon représentatif suivant le critère du secteur d'activité. Après une prise de contact par téléphone ou par mail, les entretiens se sont déroulés dans les locaux professionnels des travailleurs de façon à ce qu'ils soient dans leur environnement de travail. Six thématiques principales ont été abordées : le choix de localisation professionnelle de ces travailleurs, leurs parcours de vie, leurs activités professionnelles, leurs réseaux relationnels, leurs pratiques concrètes et leurs représentations du quartier. La réalisation des cartes mentales s'est faite à la fin de l'entretien afin qu'une relation de confiance ait été tissée entre l'enquêteur et l'enquêté. Ce choix n'est pas sans contrainte. Influencé par le contenu des échanges préalables, l'enquêté est incité à réaliser sa carte mentale comme un résumé graphique de l'entretien. La déstabilisation qui résulte de l'exercice invite toutefois à privilégier cette option ${ }^{5}$. Suivant la technique de base ${ }^{6}$ (Ramadier, 2003), la passation des cartes a été réalisée sur une feuille A4 blanche vierge avec la consigne " Dessinez votre quartier d'implantation professionnelle en y mettant les éléments importants à vos yeux ». Une prise de notes est menée en parallèle de la réalisation du dessin. La personne interrogée était ensuite invitée à commenter et expliquer sa carte, permettant ainsi «de donner une dimension réflexive au travail, en sollicitant l'enquêté sur le sens qu'il donne à son dessin » (Morange et al., 2016:168). 91 cartes ont été récoltées dont 10 sont exclues de l'analyse car elles ne comportent pas de dimension spatiale ${ }^{7}$.

9 La deuxième étape consiste en l'analyse de chaque carte mentale. Cette analyse est menée suivant trois axes : la disposition spatiale générale, l'échelle du dessin (quartier, rue, immeuble) et les éléments représentés. Les données récoltées sont codées de manière binaire $^{8}$ afin de permettre une approche statistique des cartes et des croisements avec les caractéristiques des travailleurs créatifs (secteur d'activité, date d'arrivée dans le quartier...). L'analyse des cartes s'appuie aussi sur l'étude des entretiens. Le discours des personnes interrogées est analysé de façon à saisir le sens donné aux éléments dessinés sur la carte mentale (utilisation du logiciel d'enquête qualitative Sonal).

10 La troisième étape tient à la réalisation d'une carte mentale synthétique pour chaque quartier. Il s'agit de compiler les données récoltées au niveau individuel pour mettre en évidence les représentations collectivement partagées par les travailleurs créatifs du quartier des Olivettes d'un côté et du quartier du Panier de l'autre. Pour concevoir ces cartes mentales synthétiques, tous les éléments dessinés par les personnes interrogées sont listés dans un tableur (rues, bureaux de collaborateurs...). Chaque carte du corpus est analysée suivant cette liste afin de noter la présence ou l'absence de chacun des objets suivant un code binaire ( $1=$ présence ; $0=$ absence $)$. La façon dont les éléments sont dessinés (surdimensionnement par exemple) et disposés (au centre ou non) est également prise en compte et codée. Le nombre d'occurrence(s) par élément est ensuite 
calculé. Si celui-ci est présent dans au moins $30 \%$ des cartes, il est alors intégré dans la carte synthétique. Sur cette carte, la taille des éléments ponctuels (entreprise, lieu, bâtiment...) et l'épaisseur des objets linéaires (rue, limite du quartier) varient suivant le nombre d'occurrences. Enfin, la représentation graphique, le positionnement spatial et la toponymie sont déterminés par le mode de représentation dominant dans le corpus. Les cartes synthétiques sont dessinées par le chercheur mais résultent d'une analyse des cartes mentales suivant une grille de lecture constituée de critères statistiques. Cette grille permet de réduire la part de subjectivité dans la construction des cartes synthétiques.

11 Pour le quartier des Olivettes, le corpus initial de 53 cartes mentales est divisé en deux sous-groupes traités séparément. Ce choix est apparu nécessaire à la suite de l'analyse des cartes qui a fait émerger l'existence de deux types de dessins en termes d'échelle de représentation du quartier. Dans le premier cas, le périmètre dessiné correspond à celui défini dans cette recherche (29 cartes) (Figure 1). Dans l'autre cas, le quartier dessiné se réduit à une rue et ses alentours ( 24 cartes). Deux cartes de synthèse ont donc été réalisées pour le quartier des Olivettes (Figures 3 et 4). Pour le quartier du Panier, 28 cartes mentales ont été traitées afin d'aboutir à la carte de synthèse (Figure 5). Des représentations à une échelle réduite existent également mais sont minoritaires (9 cartes) et ne justifient pas une sous-division du corpus.

\section{Entre éléments collectivement partagés et différenciations des représentations spatiales : résultats d'analyse des cartes mentales synthétiques}

12 L'analyse des cartes mentales fait apparaître sept types d'éléments dessinés : les limites $\mathrm{du}$ quartier, les voies structurantes (rue, ruelle), les places publiques, les commerces, les équipements, les autres activités culturelles et créatives et enfin les points de repères extérieurs au quartier. Parmi ces éléments, certains sont collectivement partagés par les travailleurs créatifs. Ils sont alors rendus visibles par les cartes synthétiques des quartiers des Olivettes et du Panier (Figures 3, 4 et 5). 
Figure 3. Carte mentale synthétique du quartier des Olivettes à partir des dessins à l'échelle du quartier

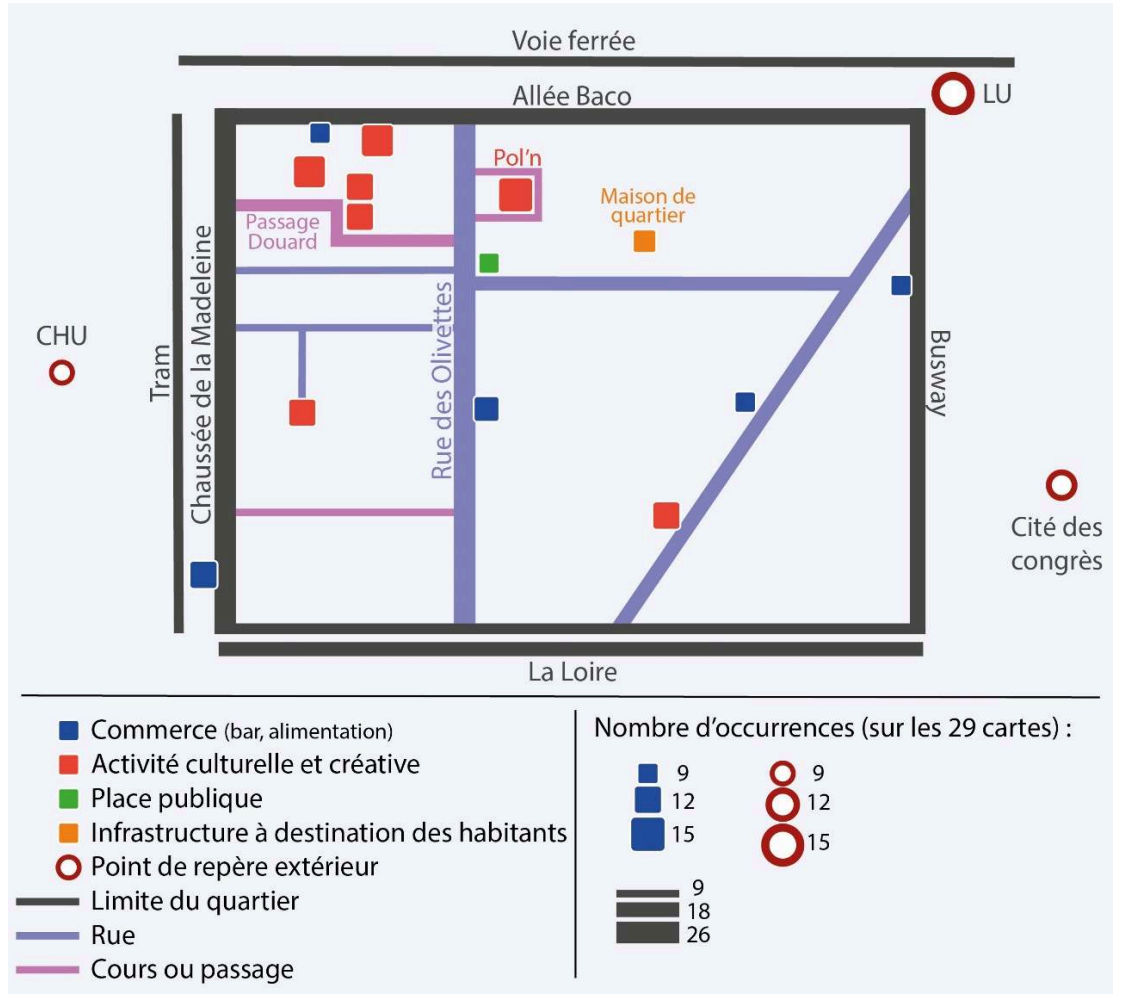

Source : Michel, 2018 
Figure 4. Carte mentale synthétique du quartier des Olivettes à partir des dessins à l'échelle de la rue

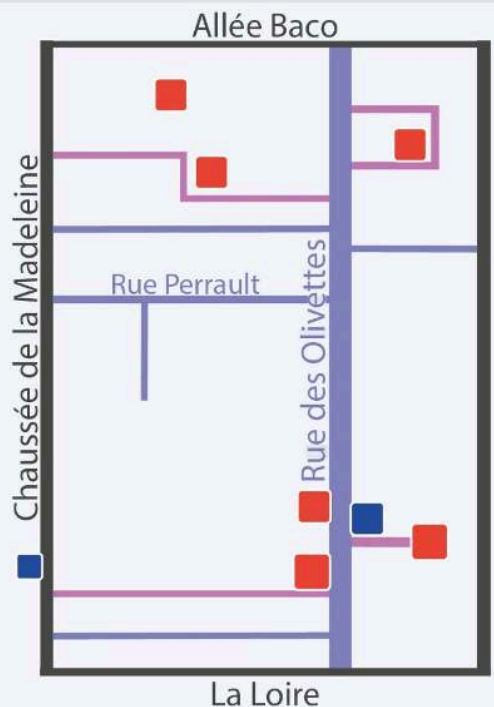

Commerce (bar, alimentation) Nombre d'occurrences

Activité culturelle et créative

(sur les 24 cartes) :

Limite du quartier

Rue

$\begin{array}{rr}7 & 7 \\ 16 & \square \\ 23 & 10\end{array}$

Cours ou passage

Source : Michel, 2018

Figure 5. Carte mentale synthétique du quartier du Panier

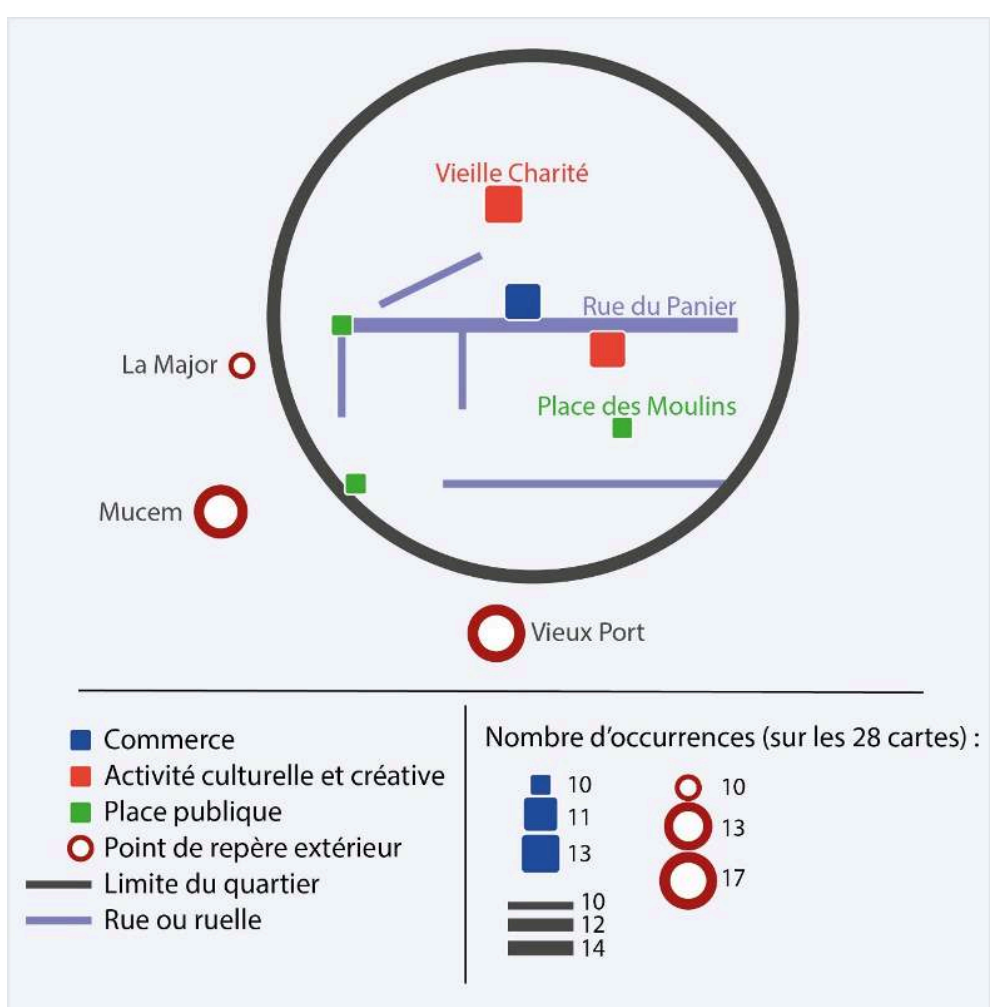

Source : Michel, 2018 
L'analyse de ces cartes synthétiques fait émerger trois résultats principaux.

\section{La dimension créative du quartier : un élément commun dans les représentations des travailleurs créatifs}

Le premier résultat tient à la mise en évidence de l'importance de la dimension créative des quartiers dans les représentations des travailleurs créatifs. La présence d'autres activités culturelles et créatives au sein du quartier fait partie des éléments mis en avant dans les dessins des travailleurs. La carte mentale d'un directeur d'association culturelle en est une illustration (Figure 6). L'utilisation de la couleur rouge symbolise sa volonté de mettre en avant la proximité de partenaires professionnels et de connaissances amicales.

Figure 6. Mise en avant de la proximité des activités culturelles et créatives dans les cartes mentales

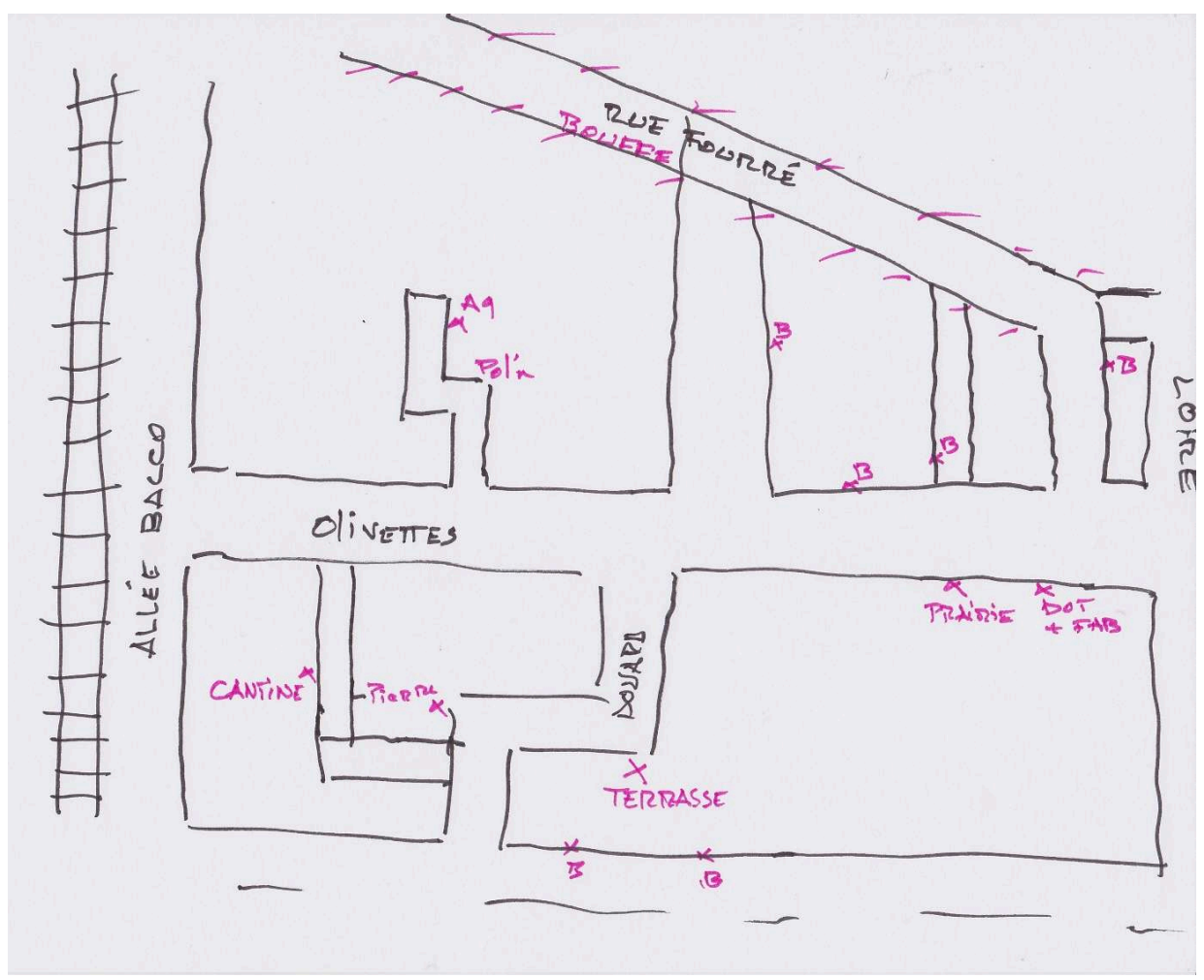

Source : carte mentale d'un directeur d'association culturelle, juillet 2015, quartier des Olivettes.

L'importance dans les cartes synthétiques des rues des Olivettes et du Panier', lieux de forte concentration de travailleurs créatifs (Figures 1 et 2), est un autre témoin de la mise en avant de la dimension créative des quartiers dans les représentations des travailleurs. La présence d'autres activités culturelles et créatives représente pour eux des opportunités de partenariats et d'échanges informels (entraide...). La proximité géographique de travailleurs appartenant aux mêmes secteurs d'activité est valorisée comme un atout pour le développement de leur projet professionnel. Elle est une ressource et un élément collectivement partagé dans les représentations que les travailleurs ont de leur quartier. 


\section{Les frontières du quartier : un élément de définition partagé par les travailleurs créatifs}

Le deuxième résultat correspond à l'identification des frontières des quartiers des Olivettes et du Panier partagées par une grande partie des travailleurs interrogés. Les limites des quartiers sont les éléments les plus dessinés après les rues des Olivettes et du Panier. Pour le quartier des Olivettes, les contours Ouest (100\% des cartes), Est (38\%), Nord (77\%) et Sud (79\%) sont fortement présents. Au Panier, toutes les limites sont présentes dans $47 \%$ des cartes. Les quartiers constituent pour les travailleurs une enclave dans la ville, sorte de village séparé du centre-ville par des frontières physiques et symboliques ${ }^{10}$. Les travailleurs créatifs mobilisent les barrières réelles entourant les quartiers des Olivettes (voie ferrée, boulevards, la Loire) et du Panier (ruptures de pente) pour définir le quartier comme une entité distincte du reste de la ville dans leurs représentations : "Au-dessus il y a Jean-Jean qui a 75 ans et au début il me disait "Oh Phil je vais à Marseille tu veux quelque chose?». Alors j'éclatais de rire mais maintenant je dis pareil quoi. On descend à Marseille, ici on n'est pas à Marseille." (Créateur installé au Panier, octobre 2015). Ce faisant, ils attribuent aux barrières réelles délimitant les quartiers le statut de frontière, entendue comme un construit social (Raffestin, 1986).

\section{Différenciations des représentations spatiales : variation des échelles du quartier}

Le troisième résultat tient à la différenciation de l'échelle spatiale de représentation du quartier par les travailleurs créatifs et nuance l'homogénéisation des représentations mise en avant par les cartes synthétiques. Les quartiers des Olivettes ( $45 \%$ des cartes) et dans une moindre mesure du Panier (32\%) font l'objet de représentations réduites spatialement. Le quartier se résume alors pour ces travailleurs à un ou plusieurs axes autour de la rue des Olivettes et du Panier (Figures 4 et 7 ). 
Figure 7. Le quartier à l'échelle d'une rue dans les représentations des travailleurs créatifs

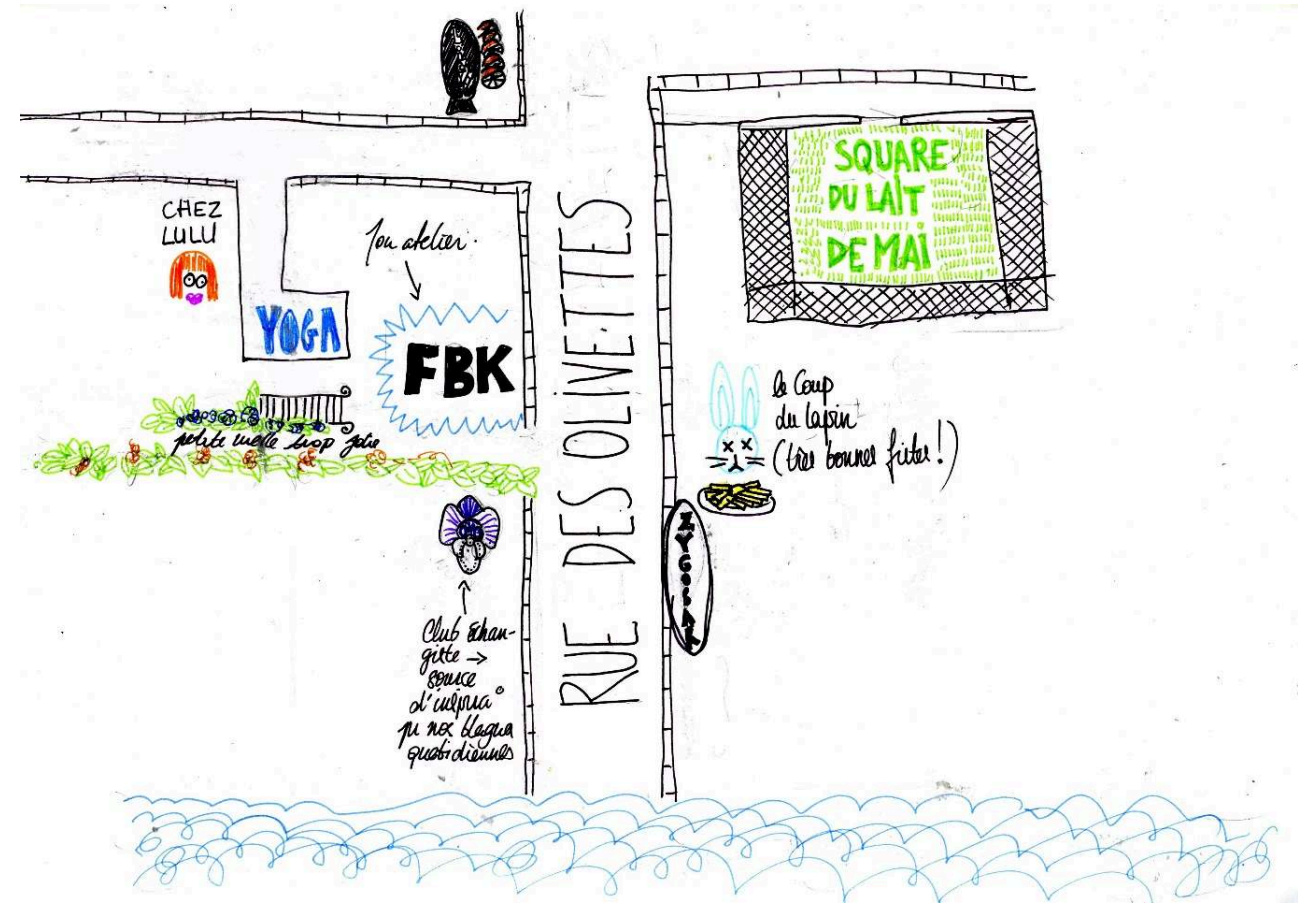

Source : carte mentale d'une graphiste, juillet 2015, quartier des Olivettes.

18 Ce type de dessins se différencie de l'échelle dominante de représentation des quartiers par les travailleurs (Figures 3 et 5). L'ancienneté dans le quartier et le secteur d'activité sont les facteurs explicatifs de la distinction entre ces deux échelles de représentation. Premièrement, les travailleurs installés depuis longtemps connaissent mieux le quartier. Ils en ont une représentation approfondie et élargie spatialement. Ainsi, les cartes dessinées à l'échelle du quartier représentent $83 \%$ des dessins pour les travailleurs arrivés avant 2010 contre $48 \%$ pour ceux arrivés après 2010. Deuxièmement, les artistes ont une représentation plus complète de leur quartier. Ils sont encouragés par la nature de leur activité à interagir avec les acteurs du quartier et à se saisir des différents lieux qui le composent pour créer des évènements culturels et de l'art urbain. Ils ne représentent que rarement le quartier à une échelle réduite (23\%) au contraire des designers dont les représentations spatiales sont centrées sur la rue des Olivettes et du Panier (78\%). Leur activité professionnelle se cantonne principalement à leur bureau et à ceux de leurs partenaires. Cela se retranscrit dans leurs représentations du quartier.

\section{Conclusion}

Les résultats présentés à partir du cas des travailleurs créatifs des quartiers des Olivettes et du Panier montrent l'intérêt des cartes mentales synthétiques dans l'identification et l'analyse des éléments collectivement partagés par un groupe social en termes de représentations spatiales. Ces cartes ont permis de mettre en évidence les éléments symboliques qui font sens au niveau collectif: la proximité d'autres travailleurs créatifs et les frontières des quartiers qui les définissent comme des enclaves distinctes du reste de la ville. La concordance des résultats obtenus aux Olivettes d'un côté et au Panier de l'autre renforce la portée de ces conclusions. Elle 
montre que ces deux quartiers proches par leur configuration géographique, leur histoire et leur dimension créative font l'objet et génèrent le même type de représentations collectivement partagées par les travailleurs créatifs qui y sont implantés.

Ces cartes synthétiques illustrent également le risque de simplification inhérent à l'exercice de synthèse. La compilation des données récoltées au niveau individuel tend à lisser les différences existantes dans les représentations des individus. L'étude préalable des cartes mentales apparaît alors nécessaire. Elle permet d'intégrer la différenciation des représentations dans l'analyse des cartes mentales synthétiques, à l'image de la prise en compte des différentes échelles spatiales de représentation dans le cas des travailleurs créatifs des quartiers des Olivettes et du Panier. Dans cette perspective, la combinaison des analyses au niveau individuel et collectif apparaît comme une piste à approfondir.

\section{BIBLIOGRAPHIE}

Attard-Maraninchi M-F., 1997, Le Panier, village corse à Marseille, Paris, Autrement.

Bailly A., 1990, « Paysages et représentations », Mappemonde, No.3, 10-13.

Depeau S., 2006, « De la représentation sociale à la cognition spatiale et environnementale : La notion de « représentation » en psychologie sociale et environnementale », Eso Travaux et documents, No.25, 7-17.

Frémont A., 1999, La région, espace vécu, Paris, Flammarion.

Gieseking J. J., 2013, « Where We Go from Here: the Spatial Mental Mapping Method and Its Analytic Components for Social Science Data Gathering », Qualitative Inquiry, Vol. 19, No.9, 712-724.

KEA, 2006, The Economy of Culture in Europe, Etude préparée pour la Commission Européenne, octobre.

Lévy J., Lussault M. (dir.), 2003, Dictionnaire de la géographie et de l'espace des sociétés, Paris, Belin. Lynch K., 1960, The Image of the City, Cambridge, MIT Press.

Morange M., Schmoll C., Toureille E., 2016, Les outils qualitatifs en géographie. Méthodes et applications, Paris, Armand Colin.

Moser G., Weiss K. (dir.), 2003, Espaces de vie. Aspects de la relation homme-environnement, Paris, Armand Colin.

Petiteau J-Y., 2012, Nantes, récit d'une traversée. Madeleine-Champ-de-Mars, Paris, Carré.

Raffestin C., 1986, «Eléments pour une théorie de la frontière », Diogène, Vol. 34, No.134, 3-21.

Ramadier T., 2003, "Les représentations cognitives de l'espace : modèles, méthodes et utilité », in Moser G. et Weiss K. (dir.), Espaces de vie. Aspects de la relation homme-environnement, Paris, Armand Colin. 
Roulier F., 2013, « Synthèses cartographiques des représentations mentales de l'espace », Mappemonde, Vol. 4, No.112, http://mappemonde.mgm.fr/num40/articles/art13403.html, consulté le 12 avril 2017.

\section{NOTES}

1. Le choix de ces quartiers est fondé sur deux critères : la concentration spatiale de travailleurs créatifs et la configuration d'ancien quartier populaire proche du centre-ville.

2. Tels qu'ils sont définis dans le rapport de KEA (2006) à la Commission Européenne, ces secteurs regroupent les arts, le patrimoine, l'audiovisuel, le jeu vidéo, l'édition, le design, l'architecture et la publicité.

3. Le quartier est défini comme une portion d'espace urbain homogène et clairement identifiable (Lévy et Lussault, 2003). Ses limites sont rendues visibles par son unité urbaine et sont généralement accentuées par des barrières physiques (cours d'eau, rupture de pente...).

4. Les travailleurs interrogés sont des femmes (44\%) et des hommes (56\%), ayant majoritairement entre 20 et 39 ans ( $56 \%)$, dirigeants de petites entreprises et associations $(2,8$ travailleurs en moyenne) des secteurs culturels et créatifs.

5. L'appréhension initiale des travailleurs créatifs face à la demande a été contournée en présentant le dessin comme une production subjective qui ne sera pas jugée. Une fois leur crainte dépassée, les travailleurs créatifs ont pris du plaisir à dessiner leur quartier et la carte mentale s'est révélée utile à l'approfondissement des discussions. Elle a participé à libérer davantage la parole de certains enquêtés justifiant son utilisation auprès des travailleurs créatifs.

6. Cette technique correspond aux cartes réalisées à main levée. Elle offre une grande liberté à la personne interrogée notamment en termes d'échelle de représentation (Morange et al., 2016).

7. Il s'agit par exemple d'une main tendue, d'une tasse de café et d'un visage souriant dessinés pour représenter la convivialité dans le quartier. La non-prise en compte de ces cartes est justifiée par l'absence de dimension spatiale. Elles constituent un matériau non exploitable pour la carte synthétique mais sont intégrées dans l'analyse globale.

8. Par exemple : représentation à l'échelle quartier $=1$; représentation à l'échelle rue ou plus réduite $=0$.

9. Dessinées dans $78 \%$ des cartes.

10. Ces éléments, et notamment l'analogie au village, sont confirmés par l'analyse des entretiens.

\section{RÉSUMÉS}

Cet article vise à proposer une démarche méthodologique de construction de cartes mentales synthétiques dont le but est de mettre en évidence les représentations spatiales collectivement partagées par un groupe social. La présentation de la méthodologie et des éléments qu'elle permet de mettre en valeur s'appuie sur les résultats d'enquêtes menées sur les représentations des travailleurs créatifs (artistes, designers...) vis-à-vis de leur quartier d'implantation professionnelle. Plus de 100 travailleurs créatifs installés dans les quartiers des Olivettes (Nantes) et du Panier (Marseille) ont été interrogés. Les cartes mentales synthétiques révèlent les représentations communes que ces travailleurs ont de leur quartier. La dimension créative des 
quartiers et les frontières qui les séparent du reste de la ville sont notamment identifiées comme des éléments collectivement partagés dans les représentations spatiales des travailleurs créatifs.

This article aims to suggest a methodological approach for the conception of synthetic mental maps. Their goal is to put forward the spatial representations that are collectively shared by a social group. The presentation of the methodology and the elements it allows to highlight is based on researches on creative workers (artists, designers...) and their representations of their professional quarter. More than 100 creative workers from the Olivettes district of Nantes and the Panier district of Marseille were interviewed. Synthetic mental maps reveal the joint representations shared by these workers. The creative dimension of quarters and the boundaries that separate them from the rest of the city are identified as collectively shared elements in spatial representations of creative workers.

INDEX

Thèmes : Carnets de terrain

Mots-clés : carte mentale, représentation spatiale, travailleur créatif, quartier urbain

Keywords : Synthetic Mental Maps, Spatial Representation, Creative Worker, Urban Quarter

\section{AUTEUR}

BASILE MICHEL

Laboratoire ESO (UMR CNRS 6590)

Université d'Angers

basile.michel@univ-angers.fr 